PRiME 2020 October 4-9, 2020
جامعة الملك عبدالله

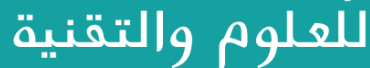

King Abdullah University of Science and Technology

\title{
Charge-Storage Mechanism of Aluminum-Sulfur Batteries
}

JASMIN SMAJIC, SHIANLIN WEE, FILIPA R. FERNANDES SIMOES, MOHAMED N.

HEDHILI, NIMER WEHBE, EDY ABOU-HAMAD, PEDRO M. F. J. COSTA

jasmin.smajic@kaust.edu.sa

Presentation \#: A02-0232

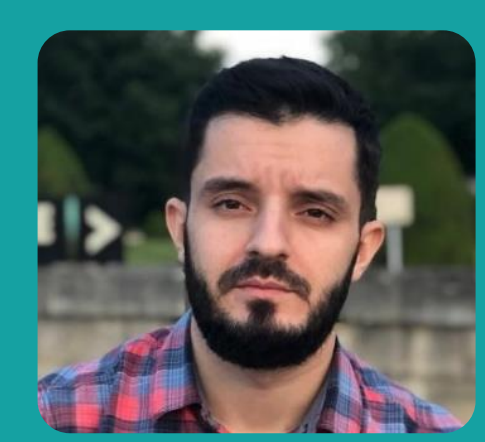

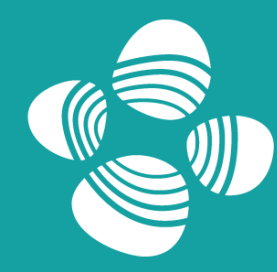
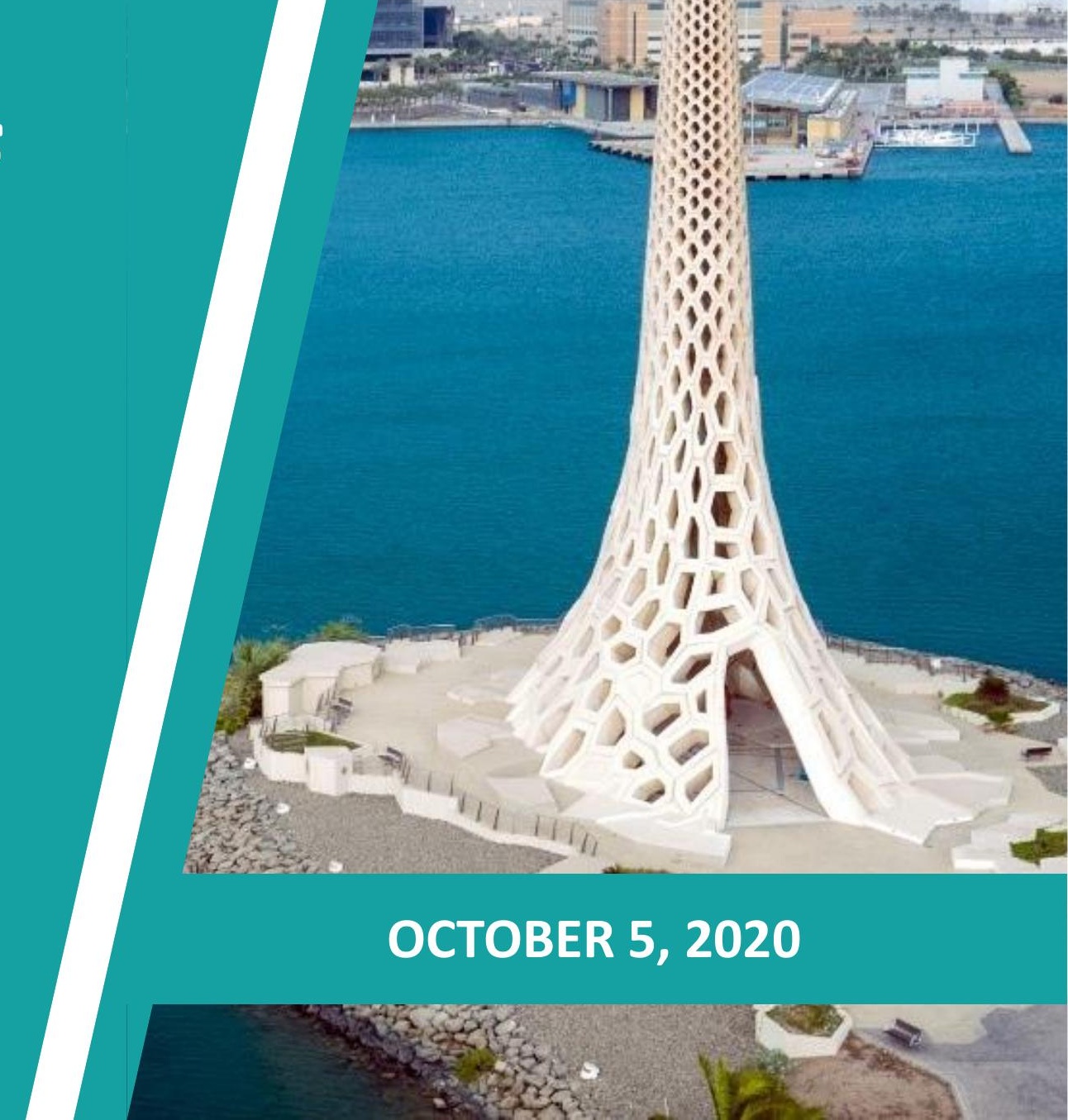


\section{Introduction}

\section{Primary energy consumption; world}
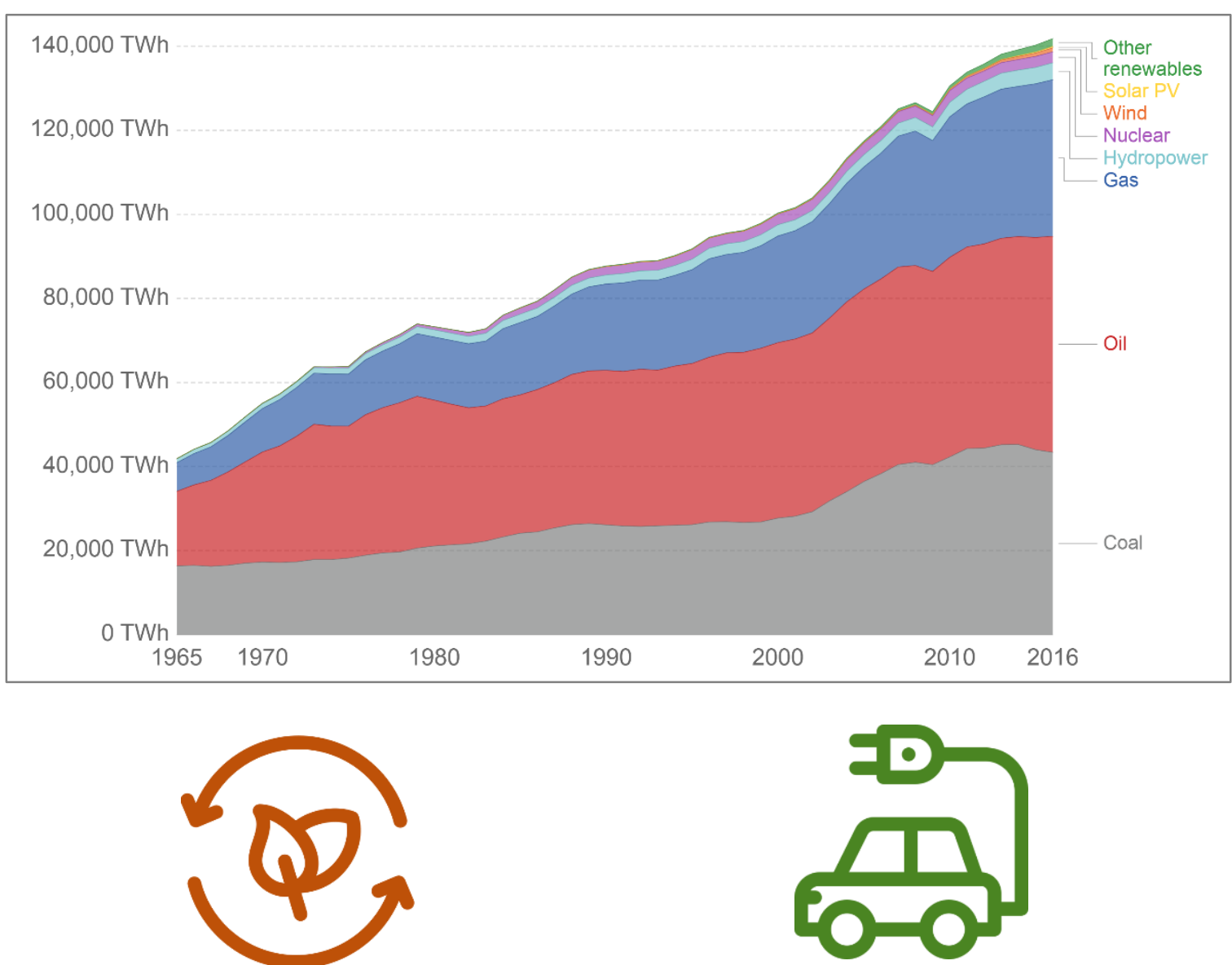

Sustainability

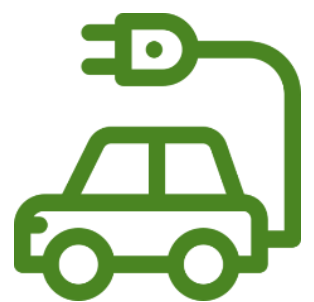

Electric vehicles
Investment in renewable energy, world
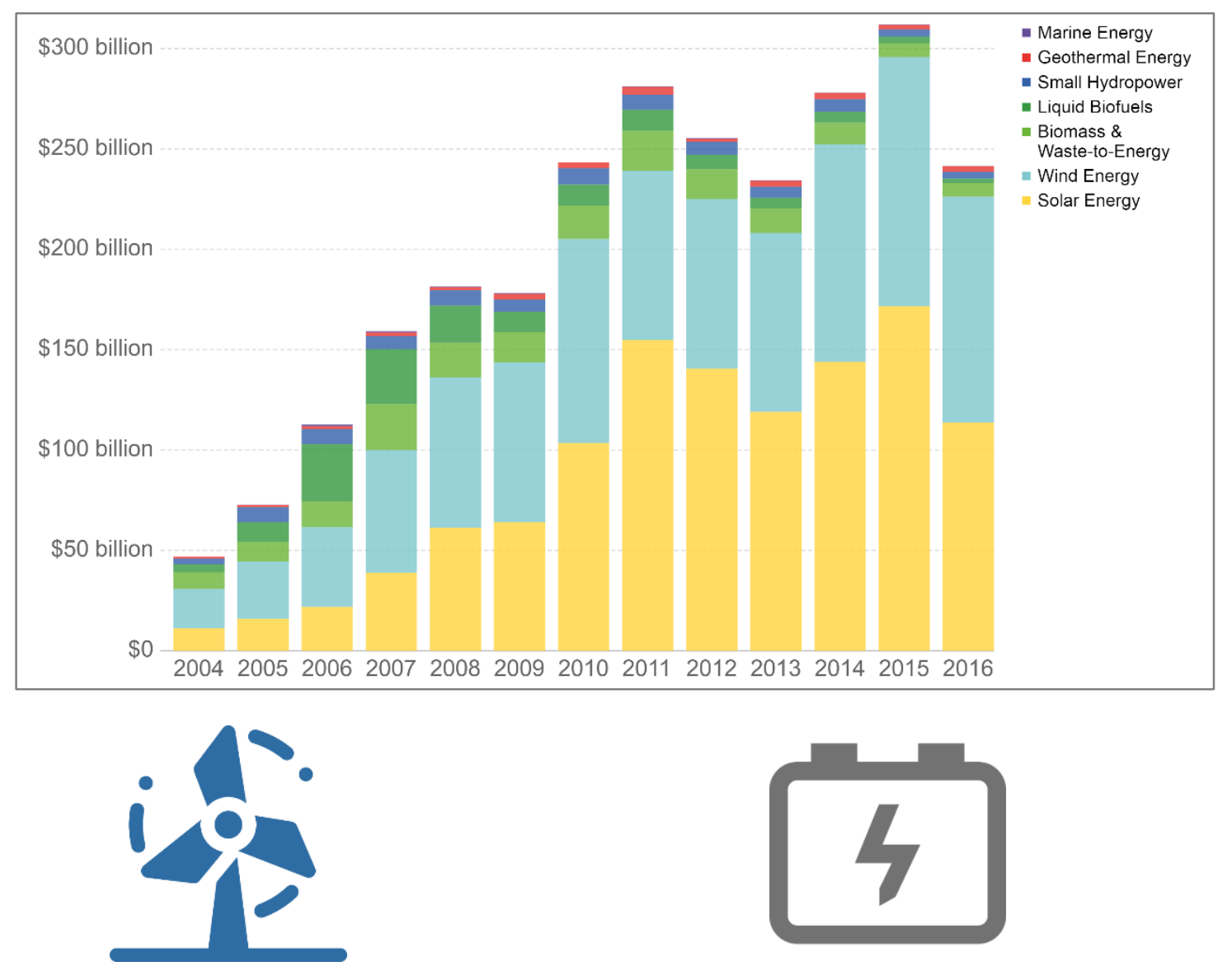

Renewable energy
Energy storage 


\section{Introduction}
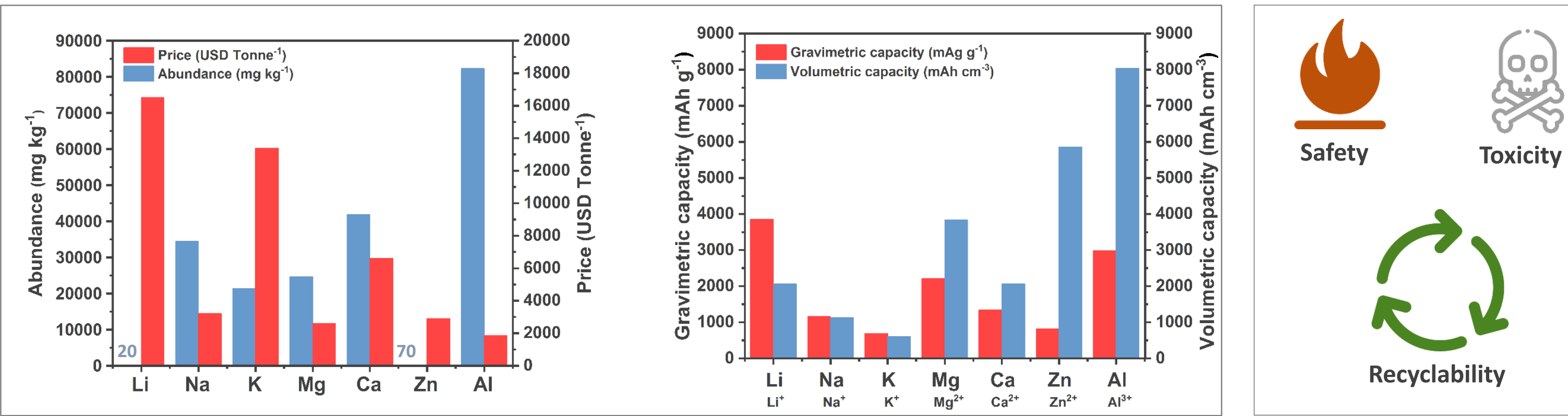

Recyclability

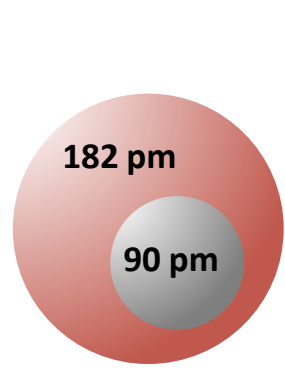

$\mathrm{Li} / \mathrm{Li}^{+}$

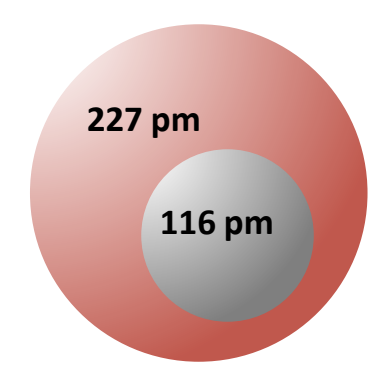

$\mathrm{Na} / \mathrm{Na}^{+}$
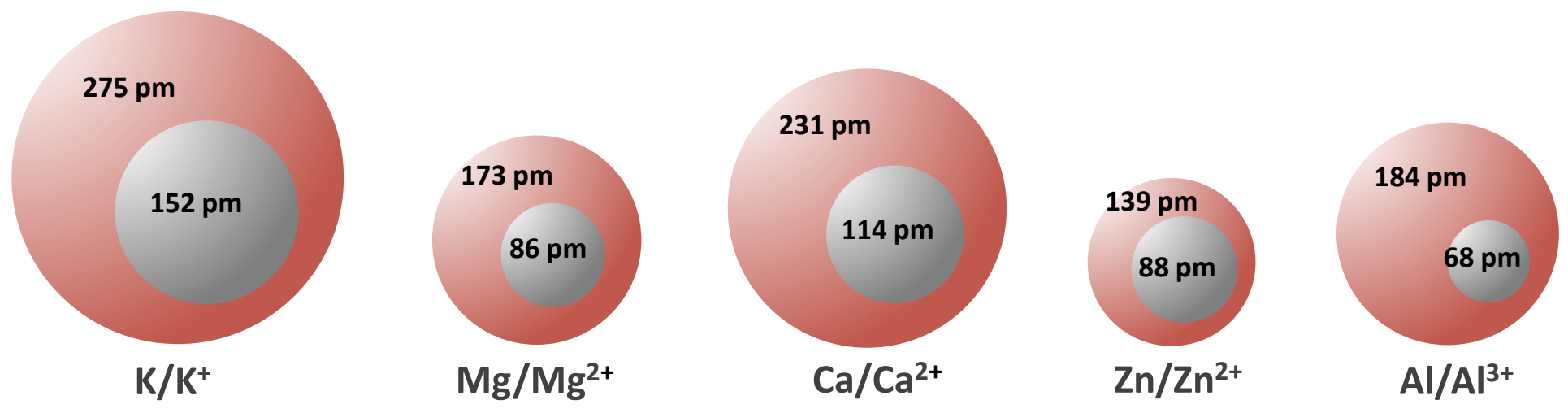


\section{Challenges}

1. Passivating surface

aluminum oxide
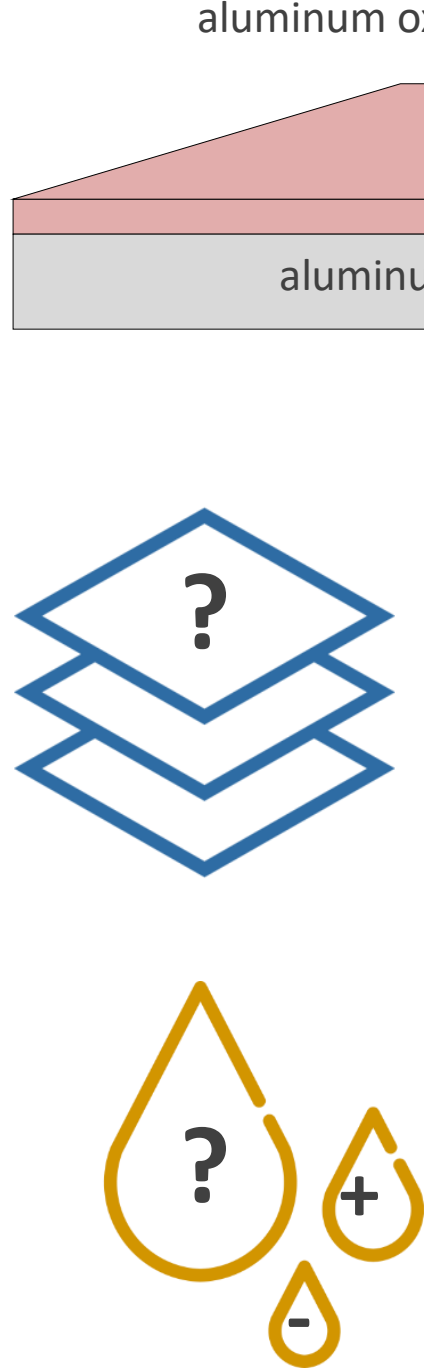

Wide band gap (6 eV) insulator

High corrosion resistance

- High density \& hardness

2. Cathode

3. Electrolyte

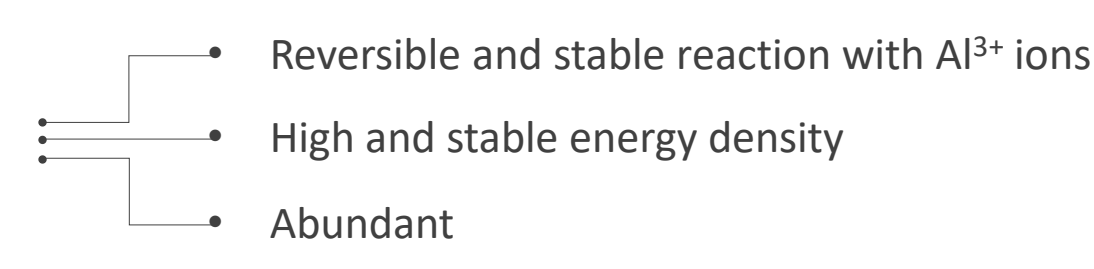

- Removes/modifies Al passivating layer

Efficient $\mathrm{Al}$ electrodeposition with $\mathrm{Al}^{3+}$ ions

- Large and stable electrochemical window

No side reactions 


\section{State of the game}

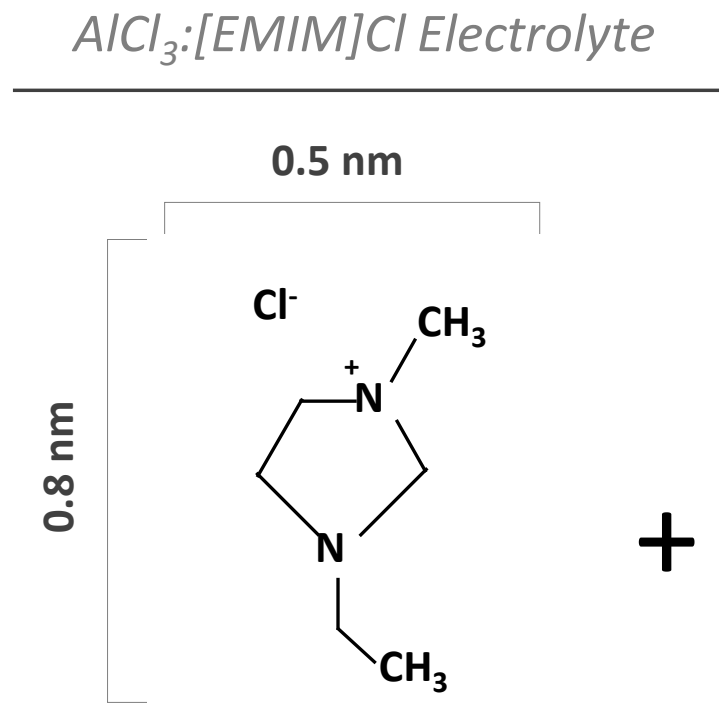

1-ethyl-3-methylimidazolium chloride

$$
\mathrm{C}_{6} \mathrm{H}_{11} \mathrm{~N}_{2} \mathrm{Cl}
$$

\section{Al electrodeposition}
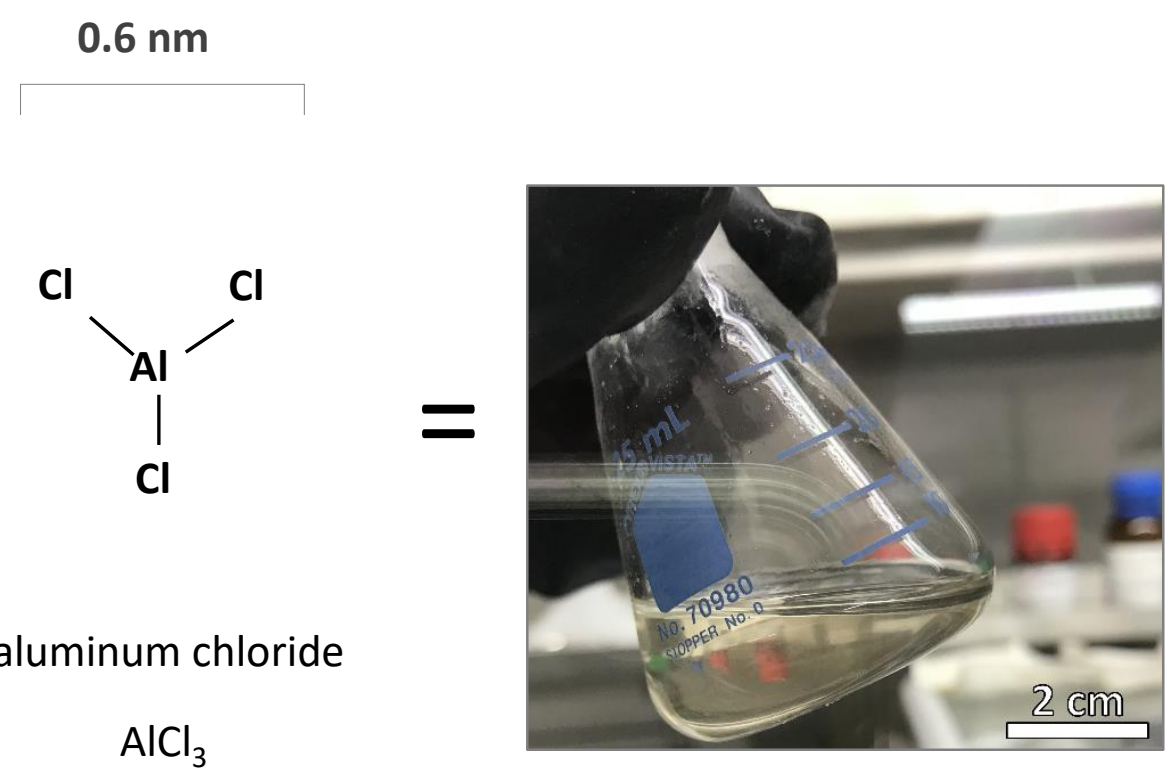

For molar ratio greater than unity

$\mathrm{AlCl}_{3}+\mathrm{C}_{6} \mathrm{H}_{11} \mathrm{~N}_{2} \mathrm{Cl} \rightarrow \mathrm{C}_{6} \mathrm{H}_{11} \mathrm{~N}_{2}{ }^{+}+\mathrm{AlCl}_{4}^{-}$

$\mathrm{AlCl}_{4}^{-}+\mathrm{AlCl}_{3} \rightarrow \mathrm{Al}_{2} \mathrm{Cl}_{7}^{-}$$$
\mathrm{AlCl}_{3}
$$

aluminum chloride

$\mathrm{AlCl}_{3}$

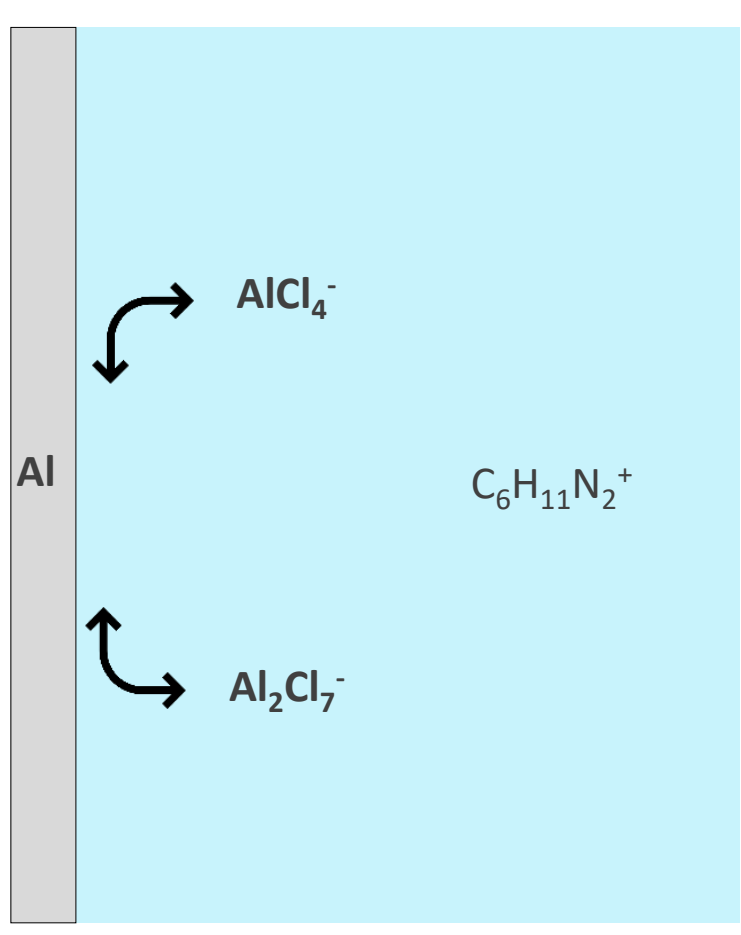

$$
4 \mathrm{Al}_{2} \mathrm{Cl}_{7}^{-}+3 \mathrm{e}^{-} \leftrightarrow \mathrm{Al}+7 \mathrm{AlCl}_{4}^{-}
$$

- No dendrite formation

- Efficient electrodeposition 


\section{Aluminum-sulfur battery}

Al-S operation

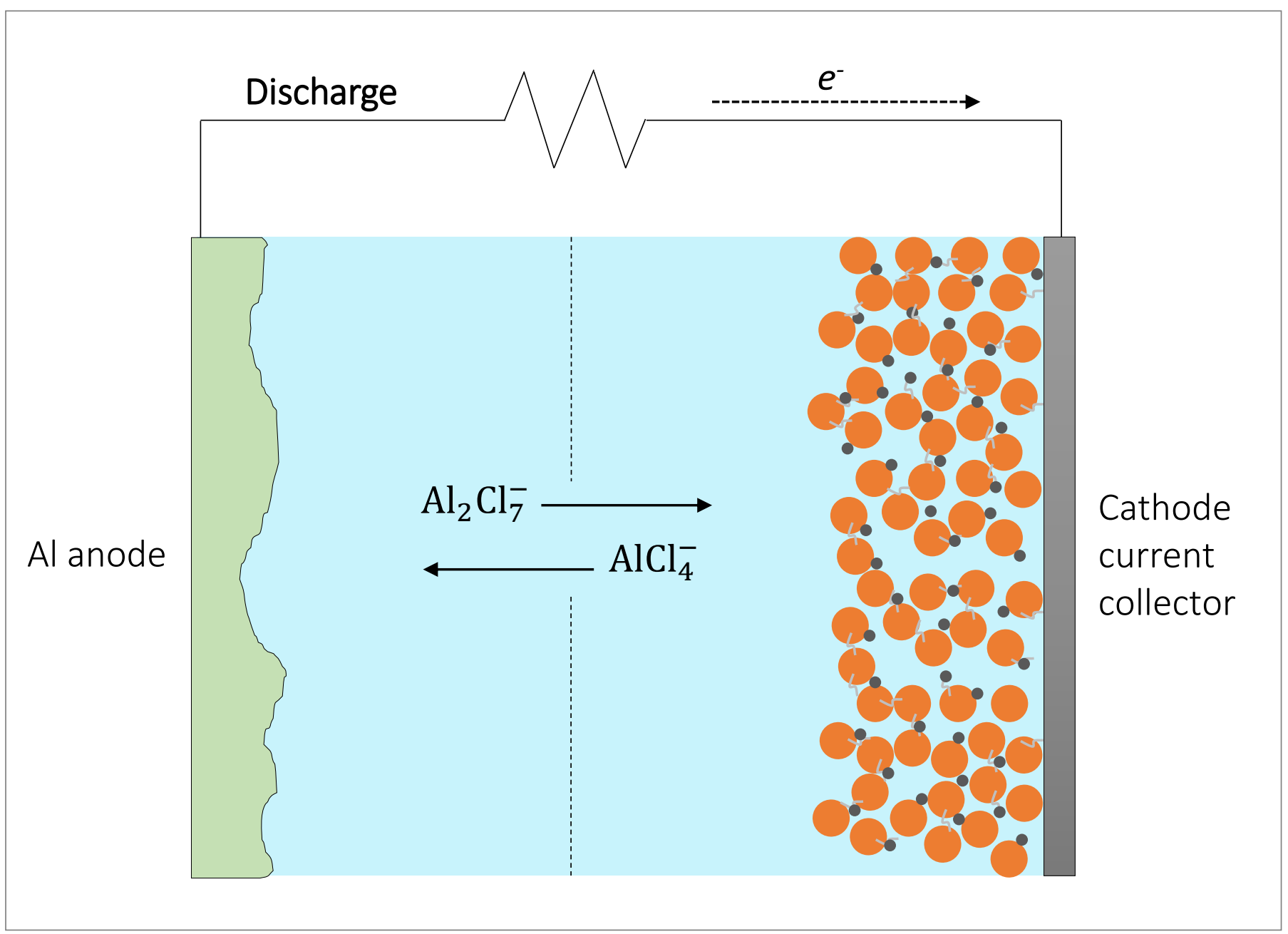

- Energy density of $\mathbf{1 3 7 1} \mathbf{W h ~ k g}^{-1}$ and $2742 \mathrm{Wh} \mathrm{L}^{-1}$

- Conversion reaction for charge storage

$$
\text { Capacity }=\frac{n F}{M}=\mathbf{1 0 7 1} \mathbf{m A h} \boldsymbol{g}^{-1}
$$

ANODE:

$$
\mathrm{Al}+7 \mathrm{AlCl}_{4}^{-} \rightarrow 4 \mathrm{Al}_{2} \mathrm{Cl}_{7}^{-}+6 \mathrm{e}^{-}
$$

CATHODE: $\quad 8 \mathrm{Al}_{2} \mathrm{Cl}_{7}^{-}+6 \mathrm{e}^{-}+3 \mathrm{~S} \rightarrow \mathrm{Al}_{2} \mathrm{~S}_{3}+14 \mathrm{AlCl}_{4}^{-}$ 


\section{Materials characterization}
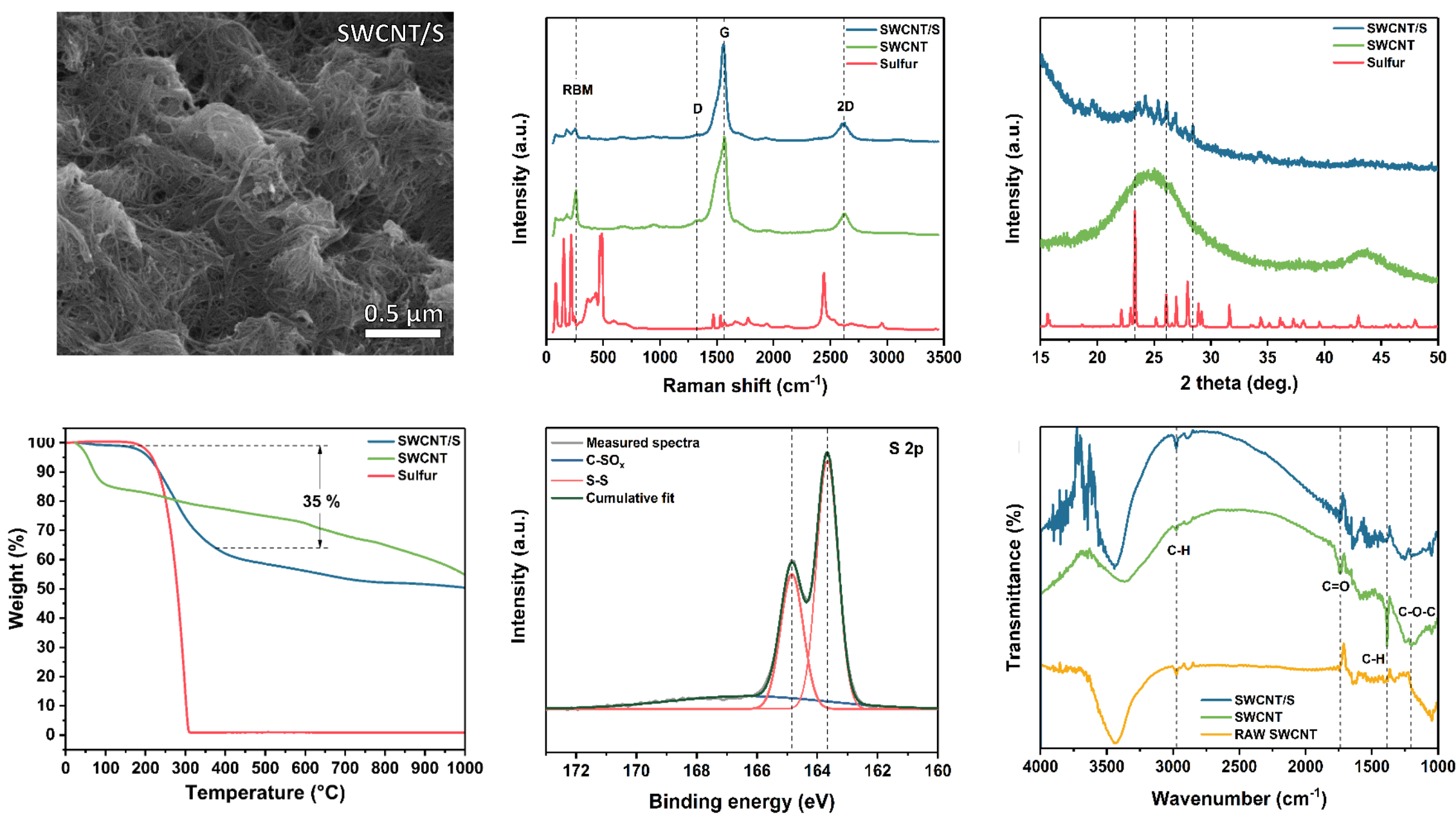


\section{Electrochemical characterization}
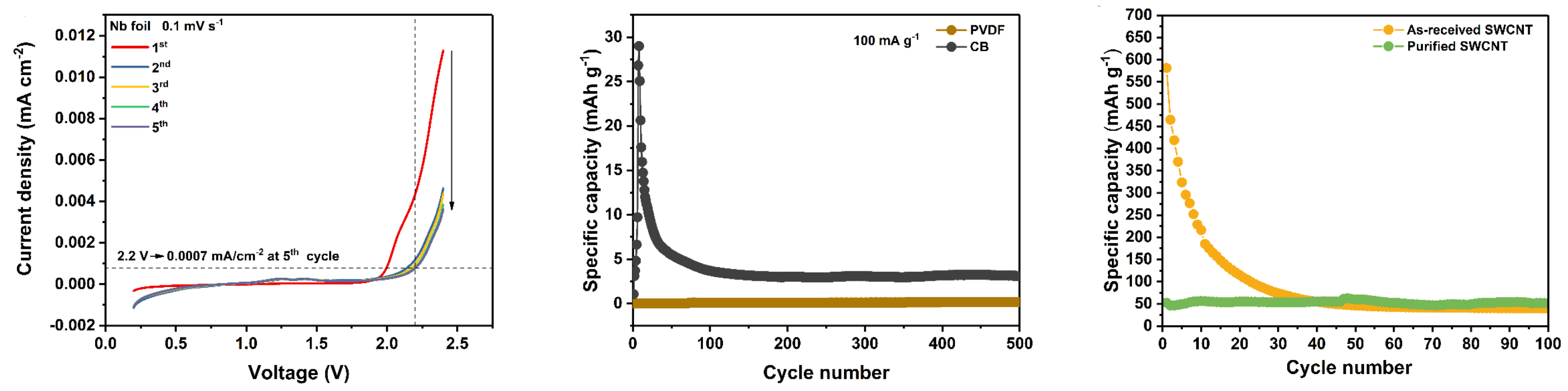

\begin{tabular}{l|l|l}
\multicolumn{3}{c}{ ICP-OES } \\
Elements & As-received & Purified \\
\hline Co & $0.7 \mathrm{wt} \%$ & $0.1 \mathrm{wt} \%$ \\
\hline Mo & $4 \mathrm{wt} \%$ & $0.7 \mathrm{wt} \%$
\end{tabular}
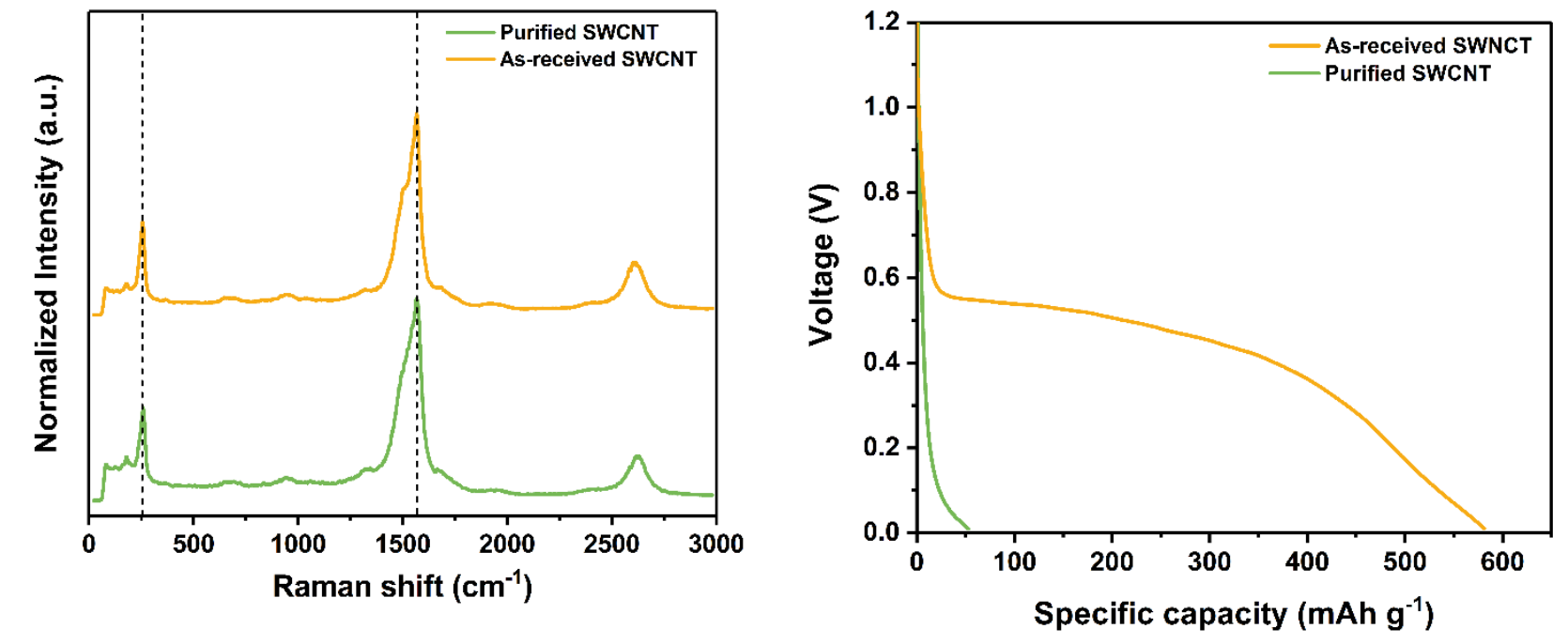


\section{Electrochemical characterization}
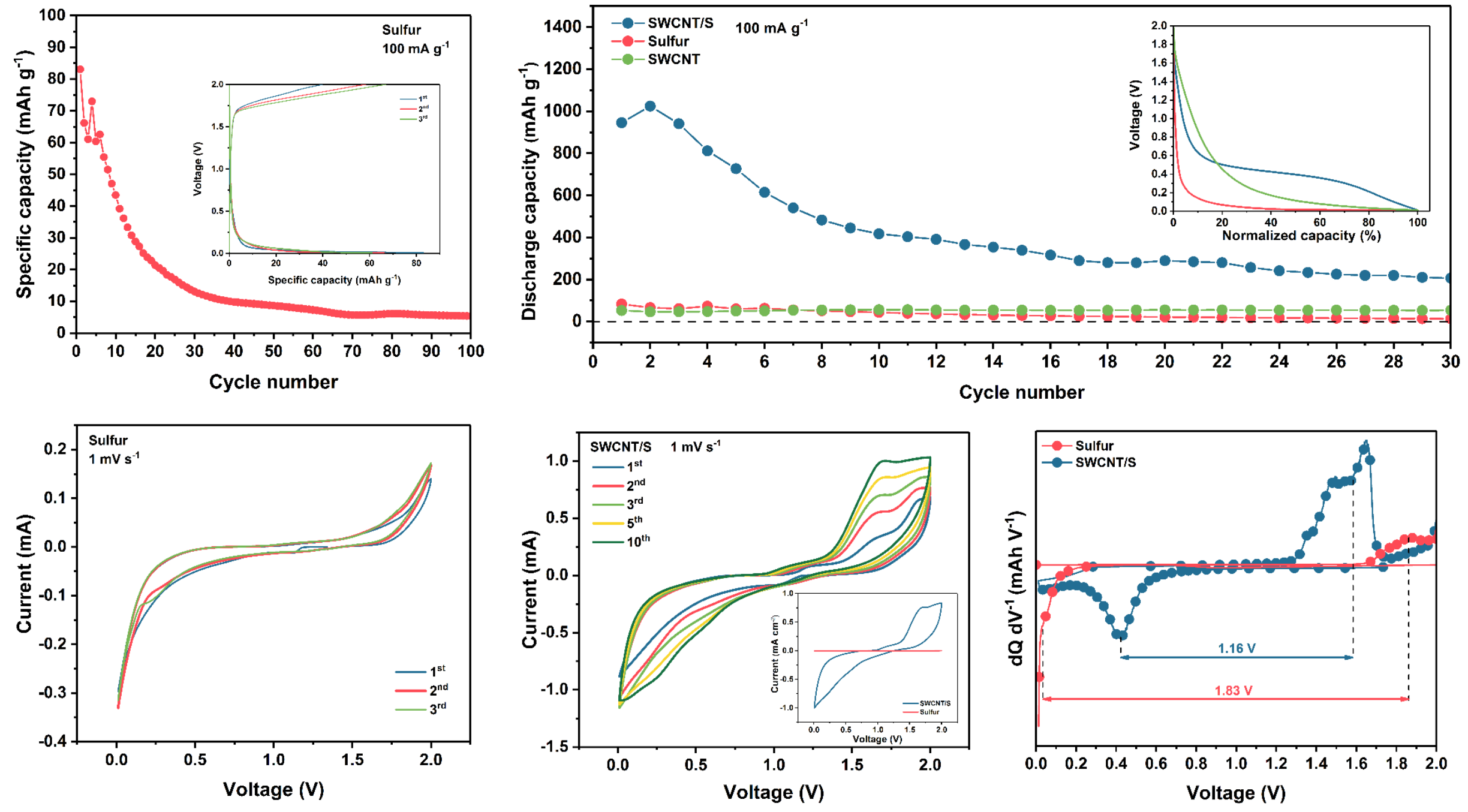


\section{Literature review}
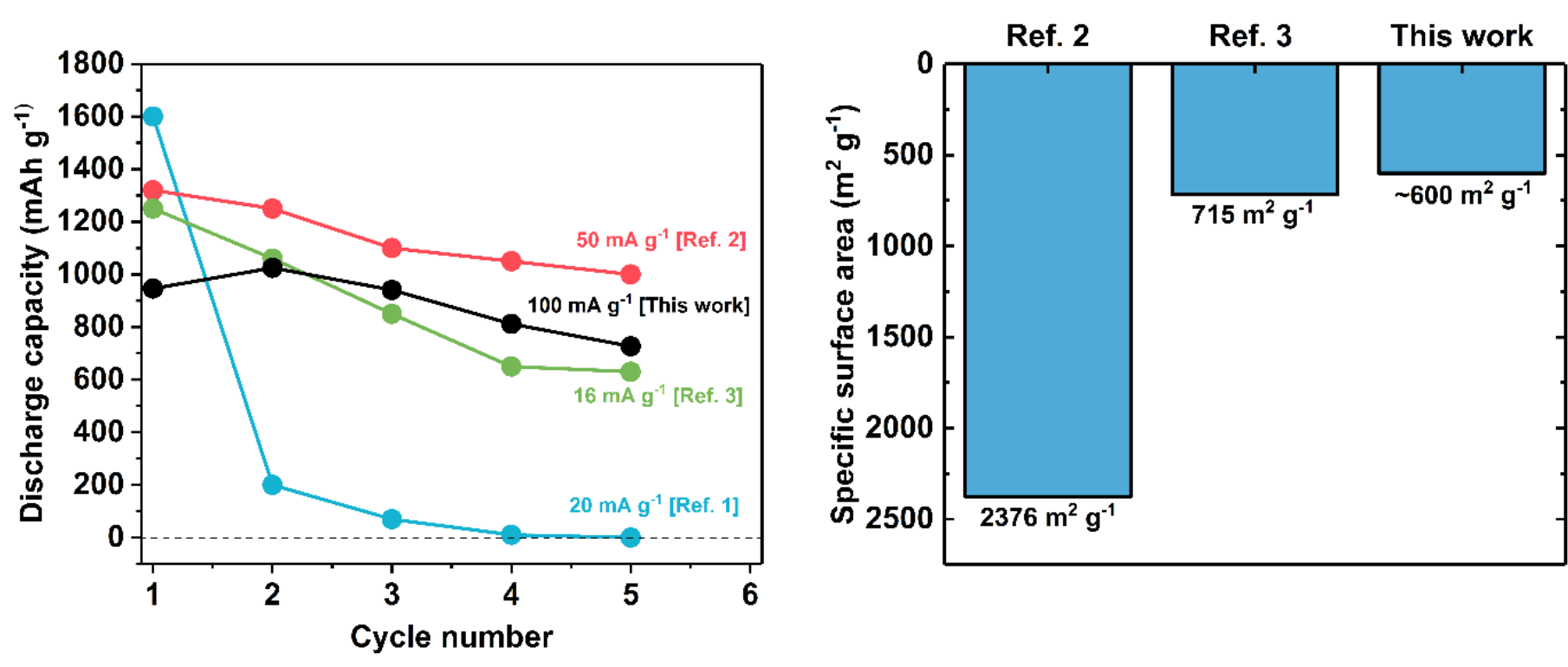

\begin{tabular}{|l|l|l|}
\hline & Carbonaceous component & Metal impurities* \\
\hline Ref. 1 & Carbon black & N/A \\
\hline Ref. 2 & Activated carbon cloth & N/A \\
\hline Ref. 3 & Carbon nanofibers, SWCNT & $<15 \% \mathrm{Fe}$ \\
\hline This work & Carbon black, SWCNT & $<0.8 \%$ Co, Mo \\
\hline *These are metal impurities present in the carbonceous materials used in the cathodes
\end{tabular}

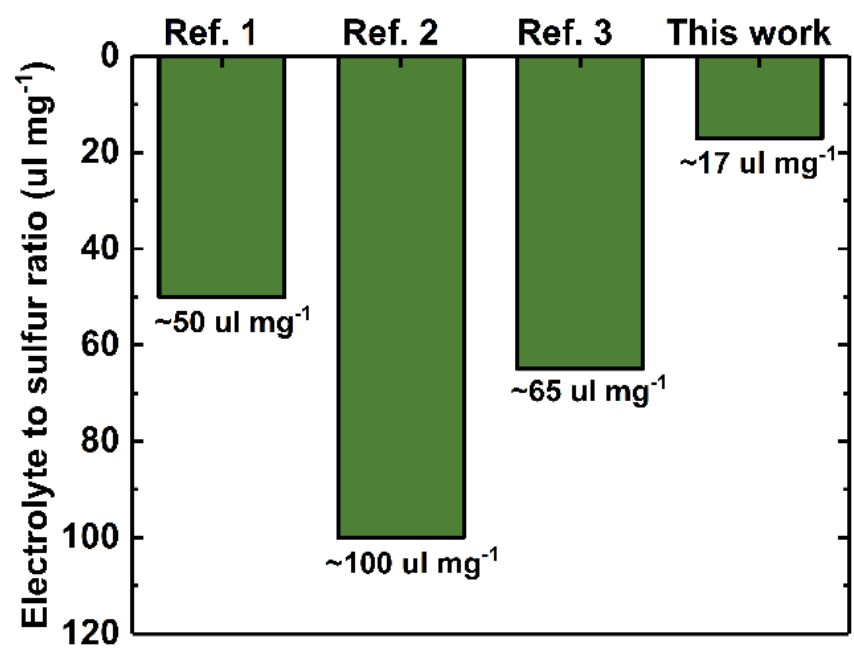

J. Maultzsch, H. Telg, S. Reich and C. Thomsen, Physical Review B, 2005, 72, 205438.

G. Cohn, L. Ma and L. A. Archer, Journal of Power Sources, 2015, 283, 416-422.

T. Gao, X. Li, X. Wang, J. Hu, F. Han, X. Fan, L. Suo, A. J.

Pearse, S. B. Lee, G. W. Rubloff, K. J. Gaskell, M. Noked and C. Wang, Angewandte Chemie International Edition 2016, 55, 9898-9901. 


\section{Electrochemical characterization}
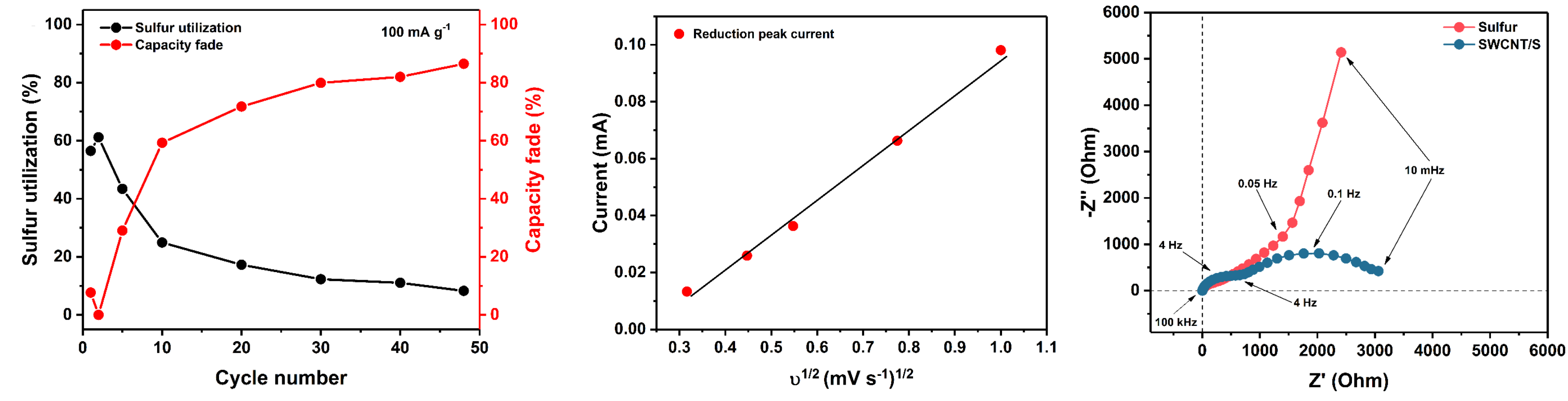


\section{Electrochemical impedance}
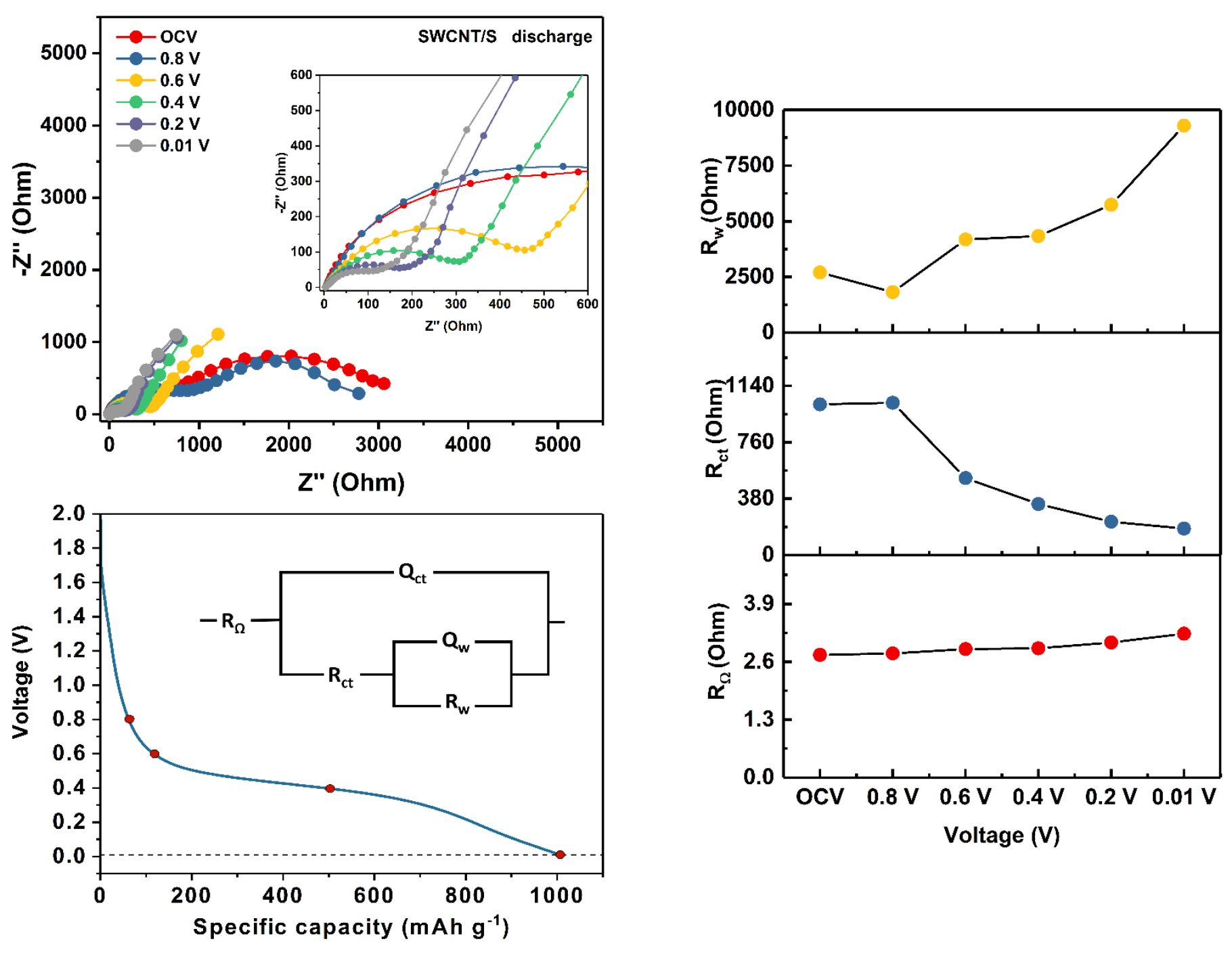


\section{Post mortem analysis}

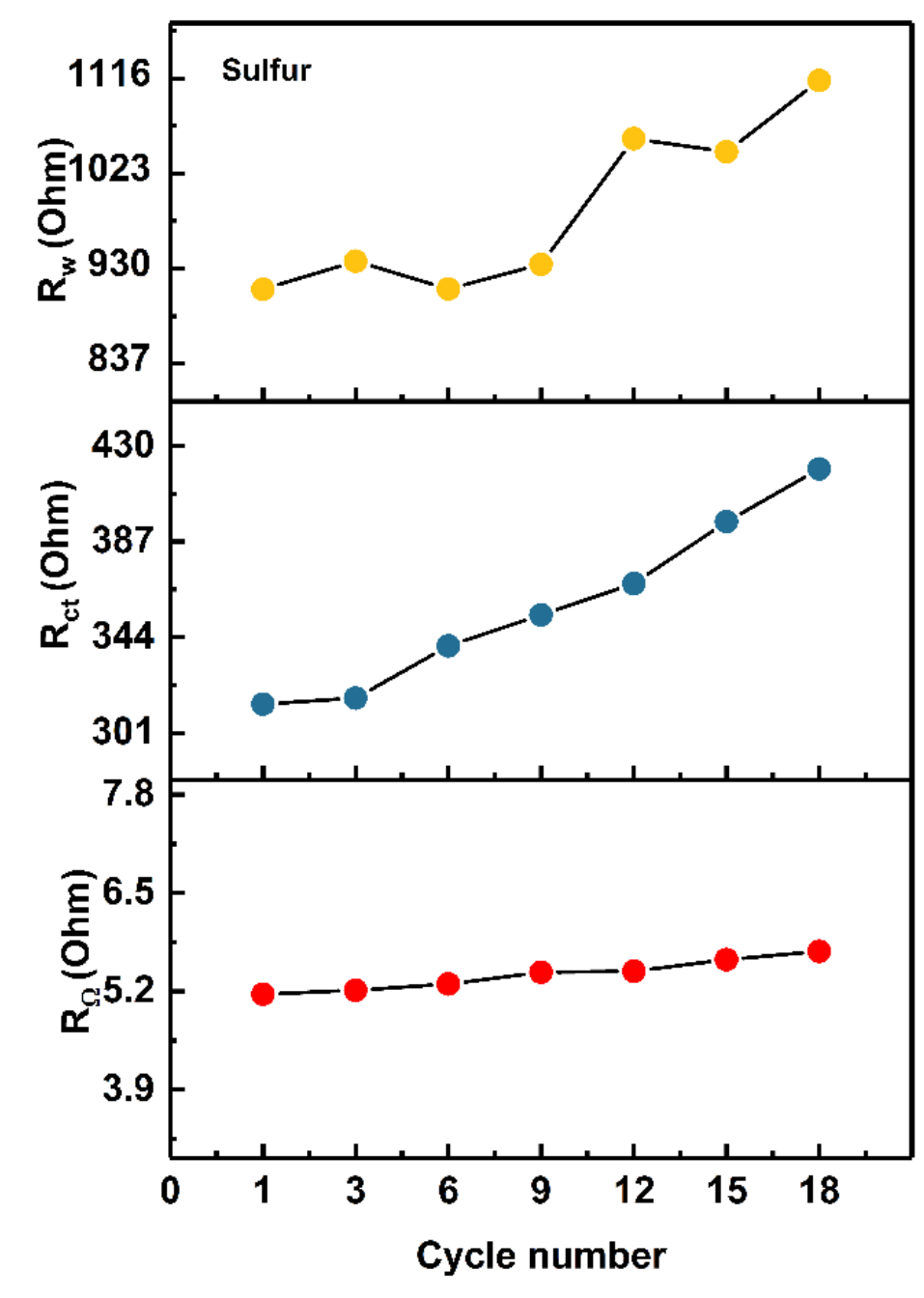

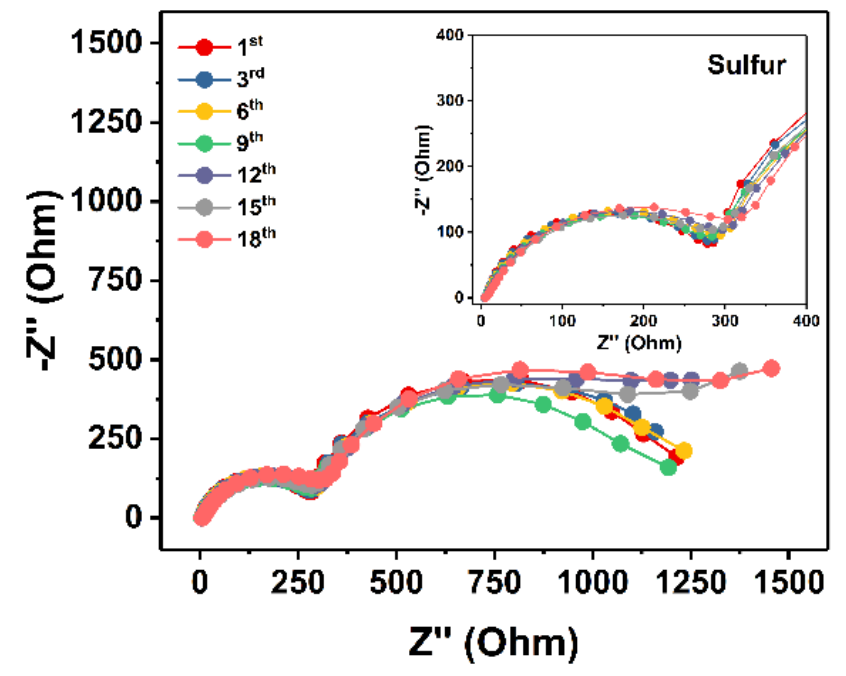

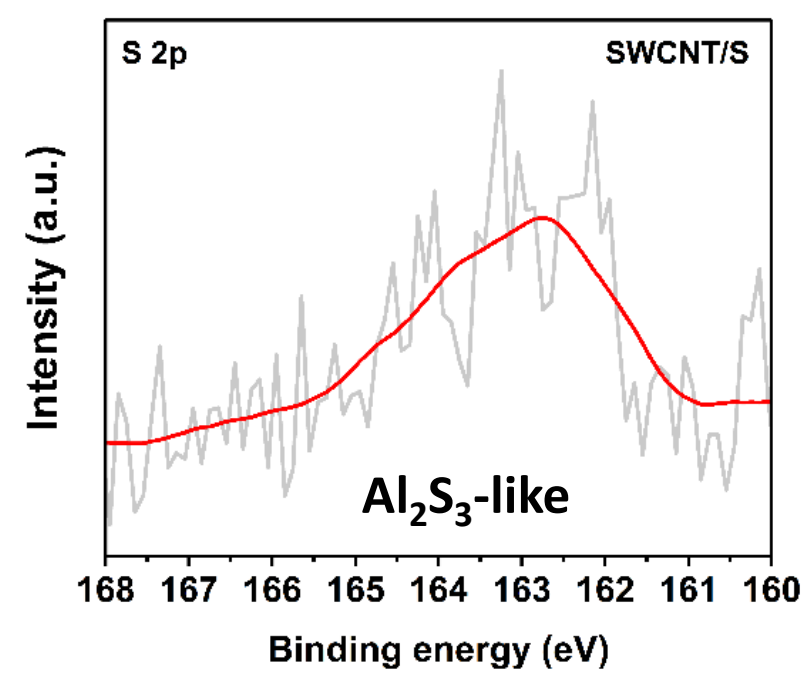

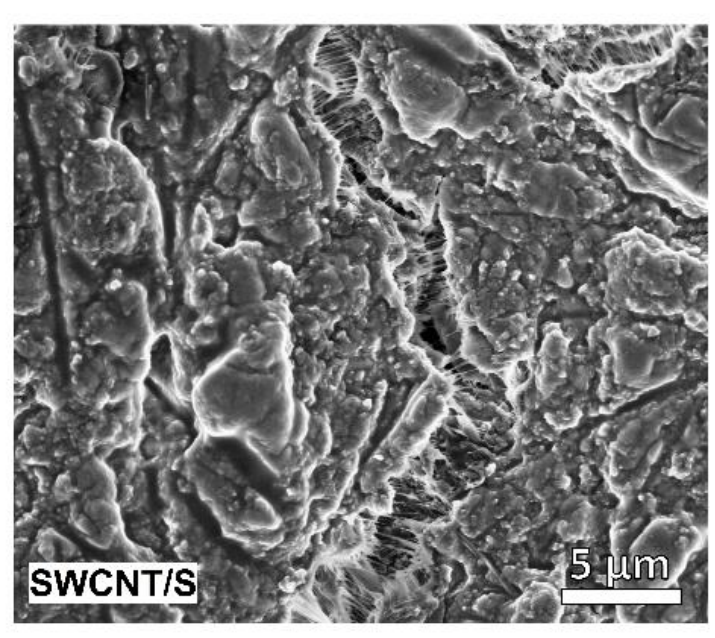

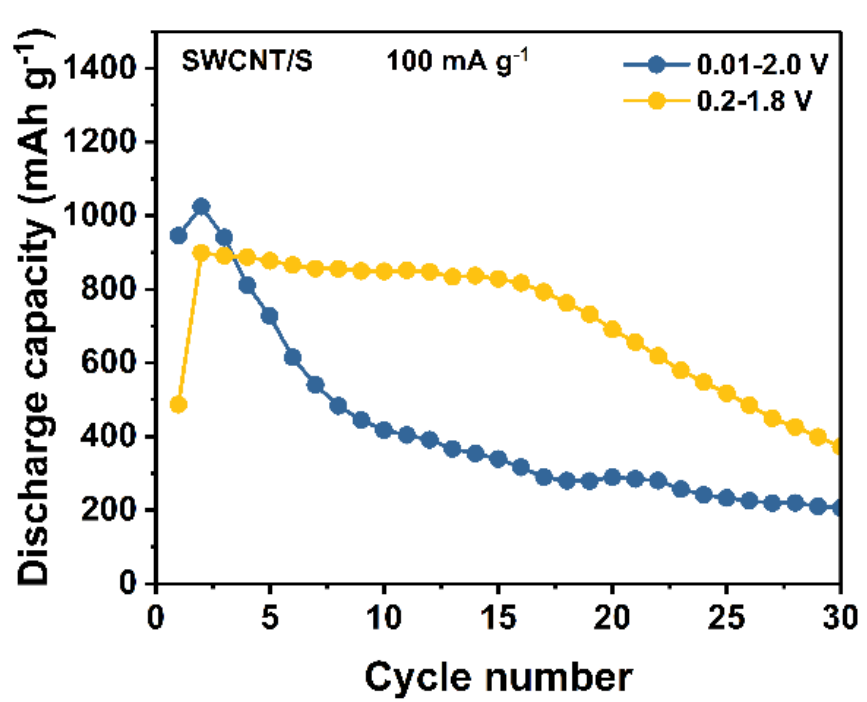


- Carbon additives can improve the cycling stability of an Al-S battery

- Capacity fade cannot be fully reversed

- Buildup of insoluble and poorly conductive discharge products, consequence of electrolyte-sulfur interaction

- New electrolytes are needed for future Al-S batteries 


\section{Acknowledgements}

- KAUST

- KAUST Core Labs
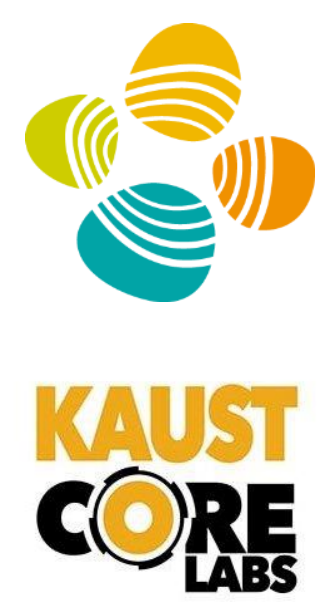

- Laboratory for Carbon Nanostructures

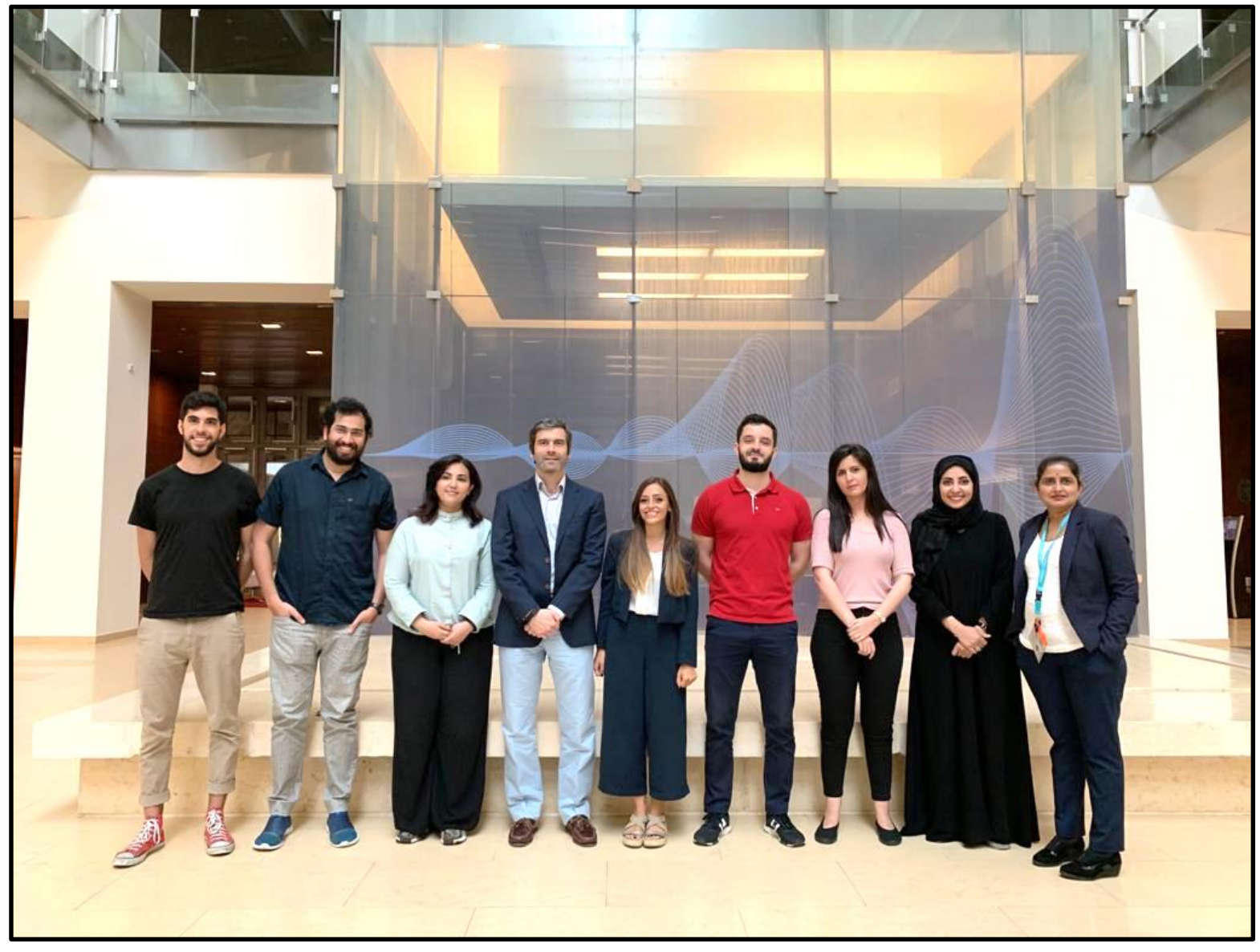

https://Icn.kaust.edu.sa/Pages/Home.aspx 
PRiME 2020 October 4-9, 2020
جامعة الملك عبدالله

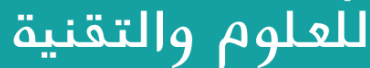

King Abdullah University of Science and Technology

\section{Charge-Storage Mechanism of Aluminum-Sulfur Batteries}

JASMIN SMAJIC, SHIANLIN WEE, FILIPA R. FERNANDES SIMOES, MOHAMED N.

HEDHILI, NIMER WEHBE, EDY ABOU-HAMAD, PEDRO M. F. J. COSTA

jasmin.smajic@kaust.edu.sa

Presentation \#: A02-0232

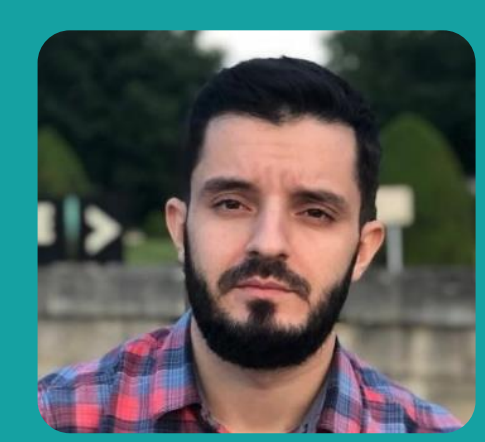

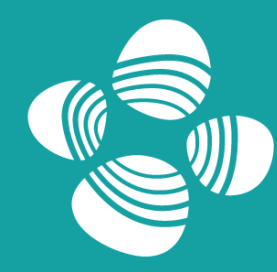
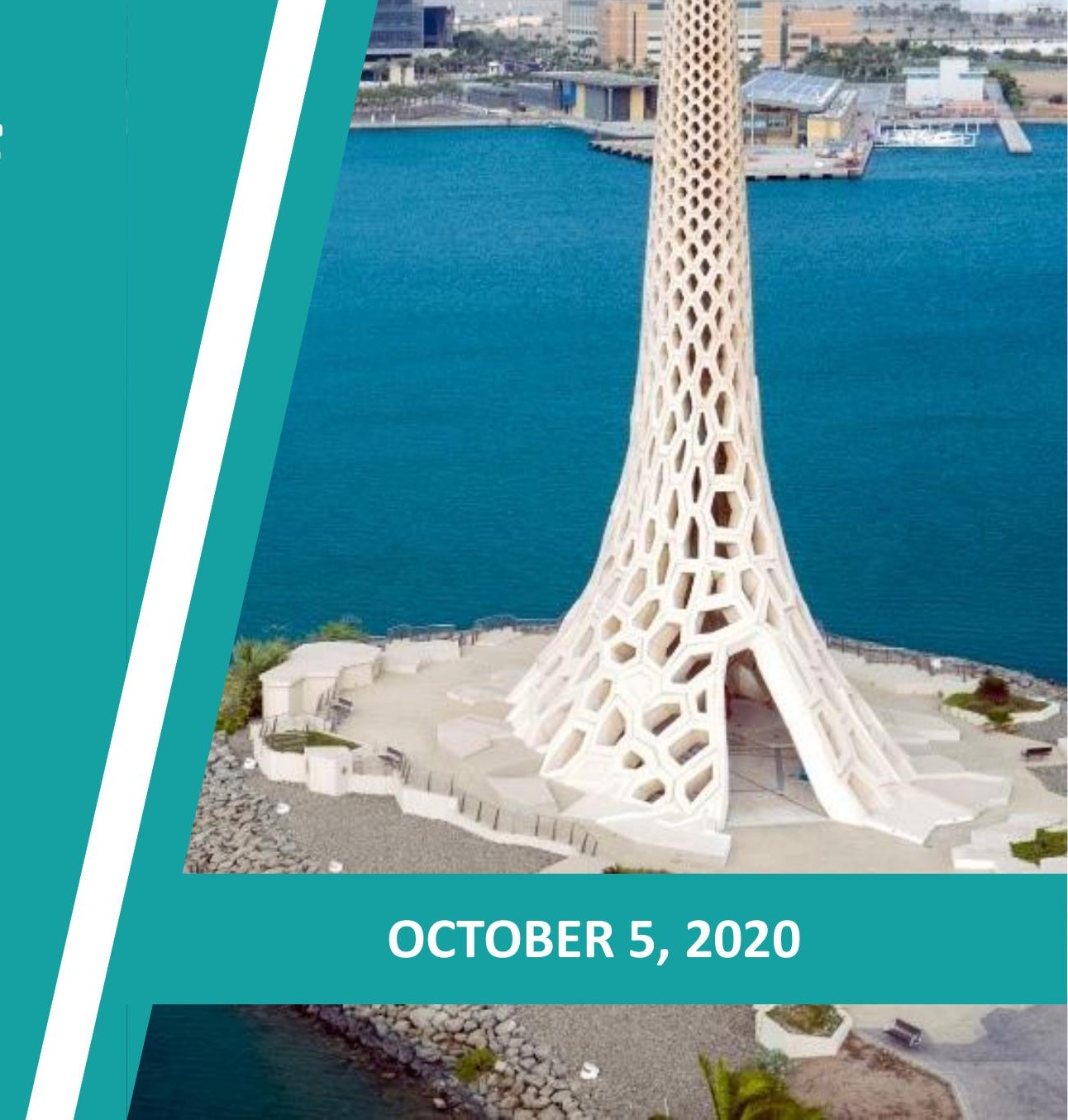